\title{
Association of vesicoureteral reflux and gastroesophageal reflux disease in children: A population-based study
}

Gregory W. Hosier ${ }^{1}$; Jeffrey P. McKay²; Heather Lynn Thomas ${ }^{3}$; Rodrigo Romao ${ }^{2,4}$; Ewa Szudek$^{4}$; Dawn L. MacLellan ${ }^{2}$

${ }^{1}$ Department of Urology, Queen's University, Kingston, ON, Canada; ${ }^{2}$ Department of Urology, Dalhousie University, Halifax, NS, Canada; ${ }^{3}$ Division of General Surgery, McMaster University, Hamilton, ON, Canada;

${ }^{4}$ Division of Pediatric Urology, Division of Pediatric Surgery, IWK Health Centre, Halifax, NS, Canada

Cite as: Can Urol Assoc J 2020 March 30; Epub ahead of print. http://dx.doi.org/10.5489/cuaj.6308

Published online March 30, 2020

$* * *$

\section{Abstract}

Introduction: Practitioners have anecdotally hinted at a possible association between gastroesophageal reflux disease (GERD) and vesicoureteral reflux (VUR). We sought to identify an association in diagnosis between GERD and VUR using a population-based dataset in a welldefined geographic area covered by a single-payer healthcare system.

Methods: A retrospective review of individuals aged 0-16 years registered in the Nova Scotia Medical Service Insurance database from January 1997 to December 2012 was completed.

Presence of GERD and VUR were ascertained based on billing codes. The baseline prevalence of GERD and VUR was calculated for this population for the same time period. Proportions of VUR patients with and without GERD were compared. The risk of being diagnosed with VUR in patients with GERD controlling for sex was calculated.

Results: Of 404300 patients identified, 6.6\% had a diagnosis of GERD ( $\mathrm{n}=27092), 0.33 \%$ had a diagnosis of VUR $(n=1348)$, and $0.08 \%$ were diagnosed with both $(n=327)$. Among patients with VUR, the prevalence of GERD was $24.3 \%$ compared to $6.6 \%$ in patients without VUR $(p<0.0001)$. Among patients with GERD, the prevalence of VUR was $1.2 \%$ compared to $0.27 \%$ in patients without $(\mathrm{p}<0.0001)$. The risk of being diagnosed with VUR was higher in the presence of GERD (odds ratio [OR] 4.49; 95\% confidence interval [CI] 3.96-5.09; $<<0.0001$ ), irrespective of sex.

Conclusions: The odds of being diagnosed with VUR is more than 4.5 times higher in an individual with GERD. The clinical significance of this association remains to be explored. 


\section{Introduction}

Vesicoureteral reflux (VUR) and gastroesophageal reflux disease (GERD) are common conditions in the pediatric population, occurring with a prevalence of $0.4-1.8 \%$ and $1-5 \%$ respectively. ${ }^{1,2}$ Both disease processes have similar timelines for presentation, occurring more commonly in early infancy and childhood, are associated with a variable spectrum of severity and often resolve spontaneously with age. ${ }^{3,4}$ Several pathophysiologic mechanisms have been described for GERD including increased duration/frequency of transient relaxation of the lower esophageal sphincter, gastro-esophageal junction incompetence, hypotensive lower esophageal sphincter, a low angle between the stomach and the esophagus, stomach factors such as small volume or poor emptying, and high abdominal pressures. ${ }^{5}$ Primary VUR consists of an abnormality of the ureterovesical junction which may include defective muscular layer of the trigone, incompetent muscle fiber architecture, and lateral displacement of the ureteral orifice and inadequate length of the intravesical ureter. ${ }^{6-8}$ High pressure voiding seen in infants has also been implicated. ${ }^{6}$ Thus, both disease processes seemingly result from a deficiency in an antireflux mechanism associated with functional rather than pure anatomical factors.

Defects in peristalsis and development have also been implicated in GERD and VUR and may share common pathophysiologic mechanisms. Interstitial cells of Cajal are a group of specialized cells found between nerves and smooth muscle fibers that act as a pacemaker for coordinating peristalsis in both the gut and ureter. ${ }^{9}$ Defects in interstitial cells of Cajal have been implicated in both GERD and VUR. ${ }^{8,10}$ Bone morphogenetic protein 4 (BMP-4) and Wingless/integrase (Wnt) signaling is known to be important for development of both gut and ureter. Abnormalities in BMP-4 and Wnt signaling have been identified in both GERD and VUR. ${ }^{11-15}$ Consequently, there is evidence for shared pathophysiologic mechanisms in GERD and VUR.

Clinical anecdotal observation suggests that GERD and VUR are concomitantly diagnosed at a higher rate than would be expected. Pooli et al (2012) explored this relationship in a selected population of 174 children and demonstrated that GERD was more frequent in patients with primary VUR. ${ }^{16}$ Since VUR is not as clinically apparent as GERD, an association between the two conditions could inform investigation algorithms for infants with a first episode of febrile urinary tract infection (UTI). We hypothesize that patients with GERD have a higher rate of VUR than those without GERD using a large administrative database.

\section{Methods}

A retrospective review of individuals registered in the Nova Scotia (NS) Medical Service Insurance (MSI) database from January 1997 to December 2012 was completed. Individuals in the province of NS, Canada must be registered in the MSI database to receive medical care paid for by the government. All claims to MSI submitted by physicians must include a diagnostic code based on the International Classification of Diseases developed by the World Health 
Organization (ICD9). All individuals aged 0 - 16 years of age registered in the NS MSI database during the time period of January 1997 to December 2012 were identified. The year 1997 was chosen as a start date as it is the first fully complete year of ICD9 billing codes in the MSI data warehouse. All individuals aged 0-16 who registered during this time period were identified. ICD9 billing codes submitted to MSI for these children for the diagnosis of VUR and/or GERD were used to identify patients of interest. Diagnostic codes for GERD have been previously described. ${ }^{17}$ ICD9 codes used for VUR were: 593.7 Vesicoureteral reflux; 593.70 Unspecified or without reflux nephropathy; 593.71 With reflux nephropathy, unilateral; 593.72 With reflux nephropathy, bilateral; 593.73 With reflux nephropathy NOS. ICD9 codes used for GERD were: 530.10 Esophagitis, unspecified; 530.11 Reflux esophagitis; 530.81 Esophageal reflux. The diagnostic code for heartburn 7871 was used for patients $>7$ years of age in keeping with the global definition of GERD consensus statement. ${ }^{18}$ Patients were excluded if they did not have diagnostic codes as described above.

De-identified data was obtained from MSI. The data was analyzed to identify individuals with a diagnosis of VUR only, GERD only, or both VUR and GERD. The baseline prevalence of VUR and GERD was calculated. Chi square testing was performed to determine if the concomitant diagnosis of GERD and VUR was greater than that expected by chance alone at a significance level of 0.05 . The risk of being diagnosed with VUR based on GERD status was further explored using logistic regression controlling for sex categories with results expressed as OR and 95\% confidence intervals.

\section{Results}

In total, 404,300 individuals age 0 -16 registered with MSI during the specified time period, and hence were included in this study. Of eligible individuals, 206,943 were male (51.2\%) and 197,357 were female (48.8\%; Table 1). VUR was diagnosed in 1,348 and GERD in 27,092 children, for an overall prevalence of $0.33 \%$ and $6.7 \%$, respectively. 327 patients were diagnosed with both conditions $(0.08 \%)$.

Overall, among patients with VUR, the prevalence of GERD was $24.3 \%$, compared to $6.6 \%$ patients without VUR $(\mathrm{p}<0.0001)$. Among patients with GERD, the overall prevalence of VUR was $1.2 \%$, compared to $0.27 \%$ in patients without GERD $(\mathrm{p}<0.0001)$. The odds of being diagnosed with VUR were higher in the presence of GERD after controlling for sex (OR 4.49; CI 3.96-5.09; $<<0.0001)$.

\section{Discussion}

Among patients with VUR, the presence of GERD was significantly increased compared to those without VUR (24.3 vs. 6.6\%). Among patients with GERD, the prevalence of VUR was significantly increased compared to those without VUR (1.2 vs. $0.27 \%)$. The differences remained regardless of sex. A link between GERD and VUR has been noted by others. In a casecontrol study of 87 children with VUR and 87 without VUR, Pooli et al found the relative risk of 
having GERD was 3.2 times higher in those with VUR. Furthermore, higher grades of VUR were associated with greater incidence of GERD. ${ }^{16}$ Our study confirms this association in a large population-based study.

Our rates of GERD and VUR were similar to other population-based studies which report prevalence of GERD in the pediatric population of $1-5 \%$ and VUR of $0.4-1.8 \%{ }^{1,2}$ In our study, approximately half of those diagnosed with GERD were age $<1$. This is consistent with other literature and may reflect the difficulty of distinguishing gastroesophageal reflux (a normal physiologic process that happens in all newborns) from GERD, which is defined as reflux that leads to troublesome symptoms and/or complications. ${ }^{2,18}$

Although the association of VUR and GERD is interesting, the clinical significance of this association remains to be explored. The diagnosis of GERD in young children is often made on clinical grounds, although invasive testing such as $\mathrm{pH}$ monitoring or endoscopy may be necessary. ${ }^{19}$ VUR is diagnosed based on a voiding cystourethrogram (VCUG). ${ }^{20}$ Based on our findings, we hypothesize that the presence of GERD increases the pre-test probability of having VUR in a child with a febrile UTI and may aid in the decision to perform invasive investigations such as VCUG. Future studies looking at the presence of GERD in children presenting with a febrile UTI who go on to have VCUG may be warranted to test the utility of GERD for predicting VUR on VCUG.

Our study has the inherent limitations of any large administrative database research. Firstly, inaccurate coding is a possibility; it is impossible to ensure that patients were categorized as having VUR only after confirmation with a VCUG. Furthermore, the diagnosis of GERD is frequently overused in the pediatric population. Secondly, it is impossible to ascertain whether patients with either diagnosis in this population were missed or not properly categorized. Finally, the association identified could be spurious or related to other confounding factors that were not accounted for.

\section{Conclusions}

Our study demonstrates the prevalence of VUR in patients with a diagnosis of GERD is higher than those without. A diagnosis of VUR is more than four times more likely in patients with GERD. While the association is interesting, the clinical significance of this association remains to be explored. 


\section{References}

1. Sargent M. What is the normal prevalence of vesicoureteral reflux? Pediatr Radiol 2000(30):587

2. Suwandhi E, Ton M, Schwarz S. Gastroesophageal reflux in infancy and childhood. Pediatr Ann 2006;35:259

3. Estrada CR, Passerottie CC, Graham DA, et al. Nomograms for predicting annual resolution rate of primary vesicoureteral reflux: Results from 2,462 children. $J$ Urol 2009;182(4):1535

4. Hegar B, Dewanti NR, Kadim M, et al. Natural evolution of regurgitation in healthy infants. Acta Paediatr 2009;98(7):1189

5. Chatila, A. T., Thu, M., Nguyen, T., Krill, T., Roark, R., Bilal, M., \& Reep, G. (2019). Natural history, pathophysiology and evaluation of gastroesophageal reflux disease.

Disease-a-Month, 1-12. https://doi.org/10.1016/j.disamonth.2019.02.001

6. Radmayr C, Schwentner C, Lunacek A, et al. Embryology and anatomy of the vesicoureteric junction with special reference to the etiology of vescioureteral reflux. Ther Adv Urol 2009;1(5):243

7. Murer L, Benetti E, Artifoni L. Embryology and genetics of primary vesico-ureteric reflux associated renal dysplasia. Pediatr Nephrol 2007;22:788

8. Arena, S., Iacona, R., Impellizzeri, P., Russo, T., Marseglia, L., Gitto, E., \& Romeo, C. (2016). Physiopathology of vesico-ureteral reflux. Italian Journal of Pediatrics, 42(1), 103. https://doi.org/10.1186/s13052-016-0316-X

9. Mostafa, R.-M., Moustafa, Y. M., \& Hamdy, H. (2010). Interstitial cells of Cajal, the Maestro in health and disease. World Journal of Gastroenterology, 16(26), 3239-3248. https://doi.org/10.3748/wjg.v16.i26.3239

10. Chen, J. (2016). Ineffective esophageal motility and the vagus: current challenges and future prospects. Clinical and Experimental Gastroenterology, Volume 9, 291-299. https://doi.org/10.2147/CEG.S111820

11. Castillo D, Puig S, Iglesias M, et al. Activation of the BMP4 pathway and early expression of CDX2 characterize non-specialized columnar metaplasia in a human model of Barrett's esophagus. J Gastrointest Surg 2012;16:227

12. Smith CM, Michael MZ, Watson DI, et al. Impact of gastro-oesophageal reflux on microRNA expression, location and function. BMC Gastroenterol 2013;13:4

13. Green NH, Nicholls Z, Heath PR, et al. Pulsatile exposure to simulated reflux leads to changes in gene expression in a 3D model of oesophageal mucosa. Int J Exp Path 2014;95,216

14. Walker KA, Sims-Lucas S, Di Giovanni VE, et al. Deletion of fibroblast growth factor receptor 2 from the peri-wolffian duct stroma leads to ureteric induction abnormalities and vesicoureteral reflux. PLoS One 2013;8(2):e56062

15. Song R, Spera M, Garrett C, et al. Angiotensin II AT2 receptor regulates ureteric bud morphogenesis. Am J Physiol Renal Physiol 2010;298(3)

16. Pooli AH, Aran S, Farhoud AR, et al. Concomitant vesicoureteral reflux and gastroesophageal reflux: An analytic cross-sectional study. Int Urol Nephrol 2012;44:32

17. Brook, R. A., Wahlqvist, P., Kleinman, N.L., Wallander, M.-A., Campbell, S. M., \& 
Smeeding, J. E. (2007). Cost of gastro-oesophageal reflux disease to the employer: a perspective from the United States. Alimentary Pharmacology \& Therapeutics, 26(6), 889-898.

18. Sherman, P. M., Hassall, E., Fagundes-Neto, U., Gold, B. D., Kato, S., Koletzko, S., ... Vandenplas, Y. (2009). A Global, Evidence-Based Consensus on the Definition of Gastroesophageal Reflux Disease in the Pediatric Population. The American Journal of Gastroenterology, 104(5), 1278-1295.

19. Vandenplas Y, Rudolph C. Pediatric gastroesophageal reflux clinical practice guidelines: Joint recommendations of the North American society for pediatric gastroenterology, hepatology, and nutrition (NASPGHAN) and the European society for pediatric gastroenterology, hepatology, and nutrition (ESPGHAN). J Pediatr Gastroenterol Nutr 2009;49(4):498

20. Subcommittee on Urinary Tract Infection. Urinary tract infection: Clinical practice guideline for the diagnosis and management of the initial UTI in febrile infants and children 2 to 24 months. Pediatrics. 2011;128(3):595 


\section{Figures and Tables}

\begin{tabular}{|l|c|}
\hline \multicolumn{2}{|l|}{ Table 1. Patients $<\mathbf{1 6}$ years old in Nova Scotia 1997-2012 (n=404 300) } \\
\hline Characteristic & $\mathbf{n ~ ( \% )}$ \\
\hline Sex & \\
\hline Female & $197,357(48.8)$ \\
\hline Male & $206,943(51.2)$ \\
\hline GERD & $27092(6.7)$ \\
\hline$<1$ & $14518(53.6)$ \\
\hline $1-5$ & $2928(10.8)$ \\
\hline$>5$ & $9646(35.6)$ \\
\hline VUR & $1348(0.33)$ \\
\hline$<1$ & $400(29.7)$ \\
\hline $1-5$ & $635(47.1)$ \\
\hline$>5$ & $313(23.2)$ \\
\hline GERD and VUR & ${ }^{*}$ \\
\hline$<1$ & $327(0.08)$ \\
\hline $1-5$ & $89(27.2)$ \\
\hline$>5$ & $165(50.5)$ \\
\hline
\end{tabular}

${ }^{*}$ Age determined at time of diagnosis with both conditions. GERD: gastroesophageal reflux disease; VUR: vesicoureteral reflux. 